\title{
PRECIPITAÇÃo PROVÁVEL PARA A REGIÃO DE MADRE DE DEUS, ALTÓ RIO GRANDE: MODELOS DE PROBABILIDADES E VALORES CARACTERÍSTICOS
}

\author{
Probable rainfall for Madre de Deus county, Alto Rio Grande region: \\ Distribution probability models and values characteristics
}

\author{
José Alves Junqueira Júnior¹, Natalino Martins Gomes², Carlos Rogério de Mello³, Antônio Marciano da Silva ${ }^{4}$
}

\begin{abstract}
RESUMO
Nos dias atuais a irrigação é uma das principais técnicas a serviço da agricultura. Entretanto, a consideração da irrigação como única fonte de suprir a demanda de água para as plantas pode acarretar em sistemas superdimensionados, o que contribui para elevar seu custo de implantação. Uma das alternativas utilizadas na solução desse problema consiste em considerar a precipitação a um determinado nível de probabilidade, ou seja, a precipitação provável, o que possibilitaria fazer a irrigação complementar. Assim, objetivou-se com o presente trabalho, caracterizar a precipitação provável na região do município de Madre de Deus, MG, comparando quatro diferentes modelos de distribuição de frequiência (Gama, Normal, Log-normal 2 e 3 parâmetros). As lâminas diárias foram totalizadas em períodos de 10, 15 e 30 dias, sendo avaliadas com 13 diferentes níveis de probabilidades, para séries históricas de 57 anos de observação, compreendido entre 1942 e 1999. Foi aplicado o teste de Kolmogorov-Smirnov a fim de avaliar a adequabilidade das mesmas e verificar qual modelo é mais adequado para cada uma das séries históricas. Observou-se que os modelos de probabilidade adequaram-se melhor ao período chuvoso, sendo a distribuição Log-normal 3 parâmetros a mais adequada para as séries históricas de período mensal e a distribuição Gama para os períodos quinzenal e decendial.
\end{abstract}

Termos para indexação: Irrigação, distribuição de freqüência, hidrologia.

\begin{abstract}
Nowadays, irrigation is one of the most important agricultural technique. Therefore, this technique can not be the only source to supply water for crops, because the irrigation system may be over designed, increasing installation costs. One of alternatives to solve this problem is to analyze the probability of rainfall, decreasing costs and easing the irrigation management. This study purposes to characterize probable rainfall for Madre de Deus Village, comparing four (4) probability distribution models (Gama, Normal, Log-normal at 2 and 3 parameters). Daily rainfall depths were totalized in consecutive periods of 10, 15 and 30 days, being evaluated for 13 different probability levels, using historical series of 57 year observation, between 1942 and 1999. KolmogorovSmirnov statistical test was applied to evaluate the adequacy of the adjusted probability models, towards to the most adequate model for each historical series. Log-normal at 3 parameters was the most adequate for monthly periods and for fortnight and decennial periods, Gama distribution was the best model.
\end{abstract}

Index terms: Irrigation, probability distribution, hydrology.

(Recebido em 10 de fevereiro de 2005 e aprovado em 16 de fevereiro de 2006)

\section{INTRODUÇÃO}

A sobrevivência dos seres vivos está ligada, entre outros fatores, à sua alimentação e, conseqüentemente à produção agrícola. A chuva, como principal fonte de água para a agricultura, tem às vezes comprometido o desenvolvimento e o abastecimento desta, fruto de sua variabilidade espacial e temporal.

O conhecimento do comportamento e da distribuição das precipitações fornece subsídio para determinar períodos críticos predominantes numa determinada região e condições de fornecer informações que visem reduzir as conseqüências causadas pelas flutuações do regime pluviométrico, seja pelo emprego da irrigação ou implantação de culturas adaptadas à sazonalidade deste regime (ANDRADE et al., 1998; GOMIDE, 1998).

A variável hidrológica precipitação é considerada contínua no espaço e no tempo, dentro da hidrologia estocástica, sendo sua ocorrência intimamente relacionada à sua freqüência (HAAN, 1979; LANNA, 2001; RIGHETTO, 1998). Desta forma, para se fazer estimativas

1Engenheiro Agrícola, Mestre - Centro Federal de Educação Tecnológica de Cuiabá/CEFET/Cuiabá - 78106-000 - Santo Antônio do Leverger, MT junqueirajunior@yahoo.com.br

${ }^{2}$ Engenheiro Agrícola, Mestre - Escola Agrotécnica Federal Antônio José Teixeira - Zona Rural, Distrito de Ceraína - Cx. P. 09 - 46430-000 Guanambi, BA - natalagricola@yahoo.com.br

${ }^{3}$ Engenheiro Agrícola, Doutor, Professor Adjunto - Departamento de Engenharia/DEG - Universidade Federal de Lavras/UFLA - Cx. P. 3037 - $37200-000$ Lavras, MG - crmello@ufla.br - Bolsista CNPq

4Engenheiro Agrícola, Doutor, Professor Titular - Departamento de Engenharia/DEG - Universidade Federal de Lavras/UFLA - Cx. P. 3037 - $37200-000$ Lavras, MG - marciano@ufla.br - Bolsista CNPq 
de sua provável ocorrência, seu estudo deve ser feito por meio de distribuições probabilísticas contínuas, sendo as mais usadas, para estudos ligados à precipitação provável, Gama incompleta, Cadeia de Markov e Log-Normal (FRIZZONE, 1979; LANNA, 2001).

A distribuição Log-Normal tem-se ajustado bem a distribuição pluviométrica brasileira e possui maior facilidade operacional quando comparadas às demais distribuições. Sampaio et al. (1999), utilizando a distribuição Log-Normal a dois parâmetros para calcular a chuva provável para Lavras, MG, observaram que esta distribuição se adequou melhor ao período chuvoso e tem se mostrado adequada para descrever a distribuição das quantidades de chuva.

Outra distribuição que tem se mostrado adequada para estudos referentes à precipitação provável, é distribuição Gama. Castro Neto \& Silveira (1981ab, 1983) estudaram, respectivamente, a precipitação provável para Lavras, MG, em períodos mensais, quinzenais e decendiais, concluindo que é possível o emprego de irrigação suplementar na região, o que pode diminuir o custo de instalação e operação de sistemas de irrigação.

A região de Madre de Deus está localizada na Bacia Hidrográfica do Alto Rio Grande, sendo banhada pelo Rio Grande. Segundo Silva \& Mello (2005), nesta região tem sido observada a implantação, cada vez maior, de sistemas de irrigação pressurizados, especialmente aspersão, com destaque para pivôs centrais em áreas de Latossolos cultivados com grãos e hortaliças. Desta forma, a geração de informações técnicas e científicas, como a precipitação provável, para subsidiar o dimensionamento dos sistemas de irrigação é de fundamental importância, especialmente em se tratando de uma região com poucas informações climáticas e hidrológicas trabalhadas de forma adequada.

Assim, objetivou-se com este trabalho: i) estudar a adequabilidade de modelos de distribuição de probabilidades normalmente aplicados ao estudo da precipitação provável, pelo teste de Kolmogorov-Smirnov; ii) determinar qual modelo de probabilidades é mais adequado para a região e para distintos períodos (mensal, quinzenal e decendial) e iii) estimar as precipitações prováveis para diferentes níveis de probabilidade.

\section{MATERIAL E MÉTODOS}

A região pertence à Bacia Hidrográfica do Alto Rio Grande, sendo este o principal curso d'água da região em estudo. A área apresenta ampla disponibilidade de recursos hídricos superficiais, com possibilidade para a utilização em agricultura irrigada, aqüicultura, abastecimento urbano e geração de energia. O clima predominante na região, pela classificação de Köppen, é transição entre Cwb e Cwa, com invernos frios e secos e verões quentes e úmidos (ANTUNES, 1986).

Os dados utilizados no presente estudo foram obtidos junto à Agência Nacional das Águas (ANA/ HIDROWEB), sendo proveniente de um posto pluviométrico instalado na região. Os dados de precipitação foram totalizados em séries mensais, quinzenais e decendiais, ajustando-se as freqüências observadas aos modelos de probabilidade, em função dos respectivos parâmetros de ajuste dos modelos, tendo sido adotada a metodologia dos momentos estatísticos, segundo Lanna (2001). A função Gama é definida pela integral:

$$
\begin{gathered}
\Gamma(\alpha)=\int_{0}^{\infty} \mathrm{x}^{\mathrm{v}-1} \mathrm{e}^{-\mathrm{x}} \mathrm{dx}, \mathrm{v}>0 \\
P(\alpha, \mathrm{x})=\int_{0}^{\mathrm{x}} \mathrm{x}^{(\mathrm{v}-1)} \mathrm{e}^{-\mathrm{x}} \mathrm{dx}
\end{gathered}
$$

A função cumulativa de probabilidade (FCP) é representada pela seguinte equação:

$$
\text { FCP }: P(x \geq X)=\frac{1}{\beta^{v} \cdot \Gamma(v)} \int_{0}^{x} u^{(v-1)} e^{-u / \beta} d u
$$

Em que $x$ representa a precipitação, $\beta$ e v parâmetros da distribuição Gama, os quais são obtidos por:

$$
\begin{gathered}
\mathrm{E}(\mathrm{X})=\beta \cdot \mathrm{u} \\
\operatorname{VAR}(\mathrm{X})=\mathrm{v} \cdot \beta^{2}
\end{gathered}
$$

E (X) e VAR (X) são, respectivamente, os momentos de $1^{\mathrm{a}}$ e $2^{2}$ ordens, dados pela média e variância amostrais.

A distribuição Log-Normal é a própria distribuição normal, quando aplicada à propriedade dos logaritmos, com sua FDP dada por:

$$
\text { FDP }: f(x)=\frac{1}{x \cdot \sigma n \cdot \sqrt{2 \pi}} \cdot \exp \left(\frac{\operatorname{Ln}(x)-\mu n}{\sigma n}\right)^{2}
$$

A estimativa da precipitação, associada a uma freqüência, é dada pela equação de Ven Te Chow, na forma logarítmica:

$$
\mathrm{X}_{\mathrm{TR}}=\exp \left(\overline{\mathrm{X}}+\mathrm{k}_{\mathrm{TR}} \cdot \mathrm{S}\right)
$$


em que, $S$. é o desvio padrão dos dados, $\overline{\mathrm{X}}$., média aritmética dos dados e $K_{T R}$, a variável reduzida da distribuição, dada pelos valores de $\mathrm{z}$, oriundos da tabela de mesmo nome.

A Função Densidade de Probabilidade (FDP) da distribuição Log-Normal a três parâmetros é representada pela seguinte equação:

$$
\begin{gathered}
\text { FDP : } f(x)=\frac{1}{(X-\beta) \cdot \sigma_{n} \cdot \sqrt{2 \cdot \pi}} \cdot e^{0,5 \cdot\left(\frac{\operatorname{Ln}(X-\beta)-\mu_{n}}{\sigma_{n}}\right)^{2}} \\
\text { sendo } x>\beta
\end{gathered}
$$

As equações para a estimativa dos parâmetros são as seguintes:

$$
\begin{gathered}
\beta=\mu_{\mathrm{n}}-\frac{\sigma_{\mathrm{n}}}{\eta_{\mathrm{y}}} \\
\eta_{\mathrm{y}}=\frac{\left(1-\phi^{2 / 3}\right)}{\phi^{1 / 3}} \\
\phi=\frac{\left[-\gamma+\left(\gamma^{2}+4\right)^{0,5}\right]}{2} \\
\gamma=\frac{[\mathrm{n}}{(\mathrm{n}-1) \cdot(\mathrm{n}-2)} \cdot \frac{\sum_{\mathrm{i}=1}^{\mathrm{n}}(\mathrm{Xi}-\mathrm{X})^{3}}{\mathrm{~S}^{3}}
\end{gathered}
$$

em que $\sigma_{n}$ e $\mu_{n}$ são, respectivamente, o desvio padrão e a média dos dados transformados e $\mathrm{S}$ é igual ao desvio padrão da amostra.

A distribuição Normal, a qual consiste de uma distribuição de dois parâmetros, possui a seguinte Função Densidade de Probabilidades:

$$
\text { FDP }: f(X)=\frac{1}{\sigma \sqrt{2 \pi}} e^{-\frac{(X-\mu)^{2}}{2 \sigma^{2}}}
$$

em que $\mu=$ média e $\sigma=$ desvio padrão.

Foi aplicado o teste de Kolmogorov-Smirnov, considerando nível de significância de 5\%, para avaliação qualitativa e adequabilidade dos modelos, comparando-se a quantidade de séries históricas que foram adequadas pelo respectivo modelo (BEIJO et al., 2003). Além disto, foram calculadas as precipitações prováveis mensais, quinzenais e decendiais, considerando níveis de probabilidades de excedência de 5, 10, 20, 25, 30, 40, 50, 60, $70,75,80,90$ e $95 \%$.

\section{RESULTADOS E DISCUSSÃO}

\section{Análise da adequabilidade dos modelos de probabilidades ajustados}

Nas Tabelas 1, 2 e 3 estão apresentados os testes de adequabilidade para as séries históricas de precipitações prováveis associadas, respectivamente, aos períodos mensais, quinzenais ( $1^{\underline{a}}$ e $\left.2^{\mathrm{a}}\right)$ e decendiais $\left(1^{\mathrm{a}}, 2^{\mathrm{a}}\right.$ e $\left.3^{\mathrm{a}}\right)$ para os quatro modelos de probabilidade ajustados. Verifica-se que a distribuição Gama foi a que melhor se adequou aos dados de precipitação para a referida região, com $65,3 \%$ das séries históricas sendo adequadas, seguido da distribuição Normal $(63,9 \%)$, Log-Normal a 2 parâmetros $(61,1 \%)$ e por último da distribuição Log-Normal a 3 parâmetros $(54,2 \%)$. Todavia, ao se analisar apenas os períodos mensais (Tabela 1), a distribuição Log-Normal a 3 parâmetros é a que apresenta o maior número de séries adequadas $(91,7 \%)$. Já para períodos quinzenais (Tabela 2), a distribuição Log-Normal a 3 parâmetros é a que apresenta a menor adequabilidade dentre os quatro modelos utilizados $(58,3 \%)$. Os demais modelos apresentaram a mesma adequabilidade $(67,7 \%)$ para o referido período, e para períodos decendiais as melhores adequabilidades foram verificadas pelas distribuições Gama e Normal, ambas com 58,3\% de séries históricas adequadas.

Ainda analisando as citadas Tabelas, na Tabela 1 verifica-se que a distribuição Normal foi a que apresentou o maior número de séries não adequadas para este período, 3 meses (junho, julho e agosto), seguida das distribuições Gama e Log-Normal a 2 parâmetros ambas com 2 meses (junho e julho) e por último a distribuição Log-Normal a 3 parâmetros com apenas um mês (julho). Isto demonstra que a distribuição Log-Normal a 3 parâmetros possui boas características para representar, probabilisticamente, séries históricas de precipitações prováveis, em períodos mensais.

Estudando-se as adequabilidades das distribuições ajustadas, para períodos quinzenais (Tabela 2), nota-se que a partir da segunda quinzena do mês de maio até a primeira quinzena de setembro, a distribuição Gama e distribuição Normal apresentaram as mesmas características, ou seja, não foram adequadas. Da mesma forma, as distribuições

Ciênc. agrotec., Lavras, v. 31, n. 3, p. 842-850, maio/jun., 2007 
TABELA 1 - Adequabilidade das distribuições de probabilidades ajustadas a períodos mensais.

\begin{tabular}{ccccc}
\hline \multicolumn{5}{c}{ Adequabilidade mensal } \\
\hline Meses & Gama & LOG-Normal 2P & LOG-Normal 3P & Normal \\
\hline JAN & A & A & A & A \\
FEV & A & A & A & A \\
MAR & A & A & A & A \\
ABR & A & A & A & A \\
MAI & A & A & A & A \\
JUN & NA & NA & NA & NA \\
JUL & NA & NA & A & NA \\
AGO & A & A & A & NA \\
SET & A & A & A & A \\
OUT & A & A & A & A \\
NOV & A & A & A & A \\
DEZ & A & A & & \\
\hline
\end{tabular}

TABELA 2 - Adequabilidade das distribuições de probabilidades ajustadas a períodos quinzenais.

\begin{tabular}{|c|c|c|c|c|}
\hline \multicolumn{5}{|c|}{ Adequabilidade quinzenal } \\
\hline Períodos & Gama & LOG-Normal 2P & LOG-NormaL 3P & Normal \\
\hline 1 & A & $\mathrm{A}$ & A & A \\
\hline 2 & A & A & A & A \\
\hline 3 & A & A & A & A \\
\hline 4 & A & $\mathrm{A}$ & NA & A \\
\hline 5 & A & A & $\mathrm{A}$ & A \\
\hline 6 & A & A & A & A \\
\hline 7 & A & A & A & A \\
\hline 8 & A & $\mathrm{A}$ & $\mathrm{A}$ & A \\
\hline 9 & A & $\mathrm{A}$ & NA & A \\
\hline 10 & NA & $\mathrm{A}$ & $\mathrm{A}$ & NA \\
\hline 11 & NA & NA & NA & NA \\
\hline 12 & NA & NA & NA & NA \\
\hline 13 & NA & NA & NA & NA \\
\hline 14 & NA & NA & NA & NA \\
\hline 15 & NA & NA & NA & NA \\
\hline 16 & NA & NA & NA & NA \\
\hline 17 & NA & NA & NA & NA \\
\hline 18 & $\mathrm{~A}$ & $\mathrm{~A}$ & A & A \\
\hline 19 & A & NA & NA & A \\
\hline 20 & A & A & A & A \\
\hline 21 & A & A & A & A \\
\hline 22 & A & A & A & A \\
\hline 23 & A & A & A & A \\
\hline 24 & A & A & A & A \\
\hline
\end{tabular}


TABELA 3 - Adequabilidade das distribuições de probabilidades ajustadas a períodos decendiais.

\begin{tabular}{|c|c|c|c|c|}
\hline \multicolumn{5}{|c|}{ Adequabilidade decendial } \\
\hline Períodos & Gama & LOG-Normal 2P & LOG-Normal 3P & Normal \\
\hline 1 & A & $\mathrm{A}$ & $\mathrm{A}$ & A \\
\hline 2 & A & A & A & A \\
\hline 3 & A & A & A & A \\
\hline 4 & A & NA & NA & A \\
\hline 5 & A & A & A & A \\
\hline 6 & A & NA & NA & A \\
\hline 7 & A & NA & NA & A \\
\hline 8 & A & A & A & A \\
\hline 9 & A & A & A & A \\
\hline 10 & A & A & A & A \\
\hline 11 & $\mathrm{~A}$ & $\mathrm{~A}$ & NA & $\mathrm{A}$ \\
\hline 12 & NA & NA & NA & NA \\
\hline 13 & NA & A & NA & NA \\
\hline 14 & NA & NA & NA & NA \\
\hline 15 & NA & $\mathrm{A}$ & A & NA \\
\hline 16 & NA & NA & NA & NA \\
\hline 17 & NA & NA & NA & NA \\
\hline 18 & NA & NA & NA & NA \\
\hline 19 & NA & NA & NA & NA \\
\hline 20 & NA & NA & NA & NA \\
\hline 21 & NA & NA & NA & NA \\
\hline 22 & NA & NA & NA & NA \\
\hline 23 & NA & NA & NA & NA \\
\hline 24 & NA & NA & NA & NA \\
\hline 25 & NA & NA & NA & NA \\
\hline 26 & NA & NA & NA & NA \\
\hline 27 & $\mathrm{~A}$ & A & NA & $\mathrm{A}$ \\
\hline 28 & A & A & NA & A \\
\hline 29 & A & A & $\mathrm{A}$ & A \\
\hline 30 & A & A & A & A \\
\hline 31 & A & NA & NA & A \\
\hline 32 & A & NA & NA & A \\
\hline 33 & A & $\mathrm{A}$ & $\mathrm{A}$ & A \\
\hline 34 & A & A & A & A \\
\hline 35 & A & A & A & A \\
\hline 36 & A & A & A & A \\
\hline
\end{tabular}

Log-Normal a 2 e a 3 parâmetros não apresentaram adequacidade para as quinzenas que se iniciaram em maio e terminaram em outubro. Verifica-se, portanto, a ocorrência de problemas para séries históricas de períodos quinzenais dos referidos meses, os quais representam o início e o final do período da seca na região de estudo. Isto pode ter ocorrido em função de uma maior variabilidade dos dados que constituem as séries, podendo-se num ano apresentar 
precipitação nula e no outro, precipitação consideravelmente elevada, dificultando sobremaneira a adequabilidade dos modelos de probabilidade, pois a estimativa dos parâmetros dos modelos de probabilidades, com base no método dos momentos, é intimamente relacionada ao comportamento da média e variância dos dados (WALPOLE \& MYERS, 1978).

Para períodos decendiais (Tabela 3), observam-se características semelhantes ao do período quinzenal. Observa-se que a partir do terceiro decêndio de abril até o terceiro decêndio de agosto, a distribuição Gama e distribuição Normal apresentaram as mesmas características de não adequabilidade. As distribuições Log-Normal a 2 e 3 parâmetros, também apresentaram características semelhantes às do período quinzenal, com os períodos de não adequacidade iniciando-se no primeiro decêndio do mês de fevereiro e terminando no segundo decêndio do mês de novembro. Um fato que deve ser destacado é que para períodos decendiais, a quantidade de séries adequadas foi consideravelmente menor que para os períodos quinzenais e mensais.

A adequabilidade dos modelos de probabilidades utilizados, segundo o teste de Kolmogorov-Smirnov, diminuiu com o tamanho dos períodos analisados, ou seja, a possibilidade de um dos modelos utilizados ser adequado para o período decendial foi sempre menor que para períodos quinzenais e estes menores que períodos mensais. Isto se deve ao fato de que ao diminuir os períodos de análise aumentou-se o desvio padrão e conseqüentemente o coeficiente de variação da amostra. Esta situação implica em maior dificuldade de se obter adequabilidade do modelo de probabilidades, pois será estimada uma freqüência teórica influenciada pelos parâmetros dos modelos de probabilidades, e esta será muito distorcida em relação à freqüência observada, uma vez que esta última não depende das características estatísticas da amostra.

\section{Precipitação provável estimada para cada período de análise}

Os valores estimados para as precipitações prováveis mensais, associadas aos níveis de probabilidades de 75, 80, 90 e $95 \%$, estão destacados na Figura 1. Utilizou-se a distribuição Log-Normal a 3 parâmetros por esta ter sido mais adequada para a respectiva série. Observa-se que a região de Madre de Deus apresentou bons valores de precipitação mensal para

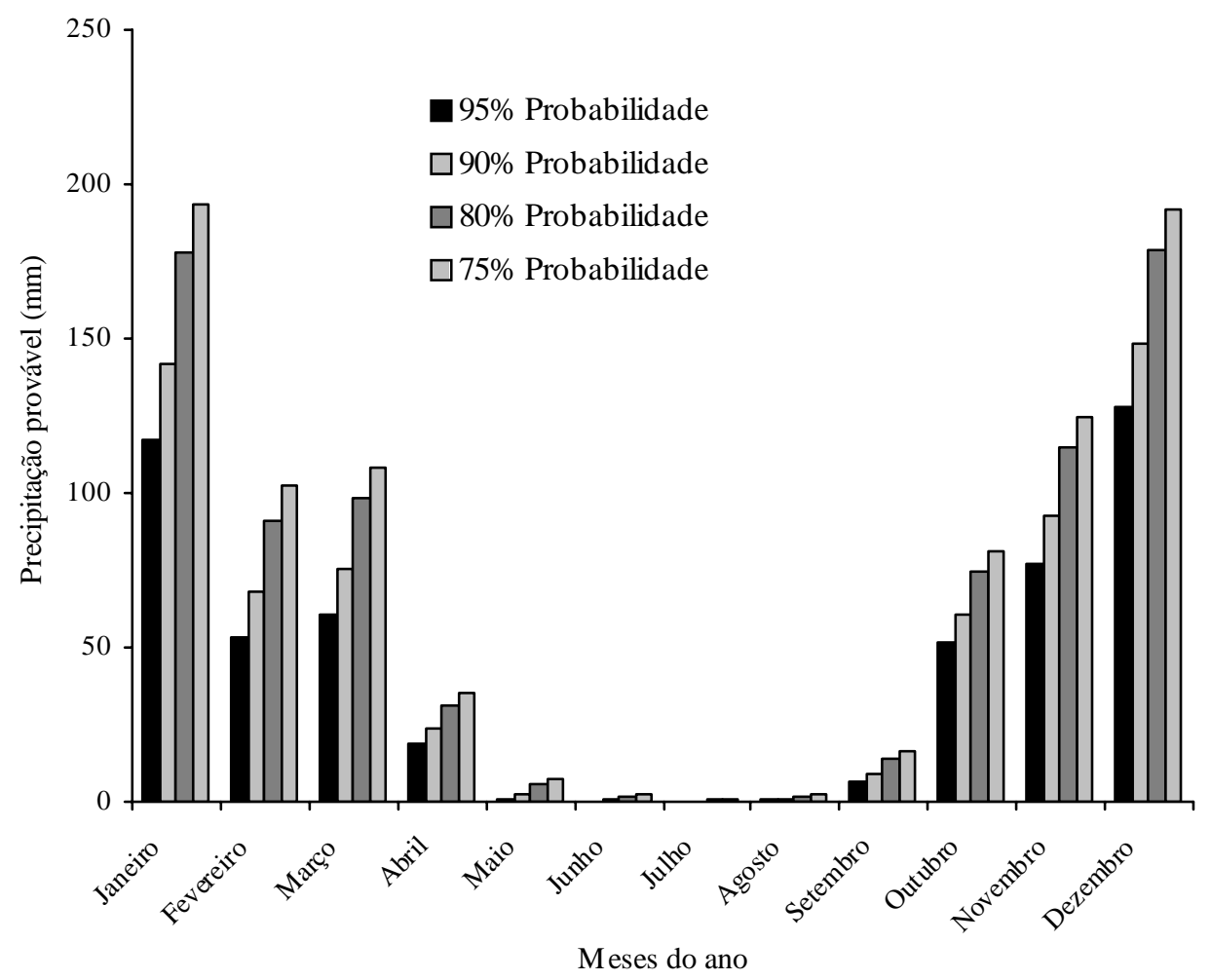

FIGURA 1 - Precipitação provável mensal segundo a distribuição Log-Normal 3 parâmetros. 
os maiores níveis de probabilidade de excedência, mostrando ser uma região onde devem ser pequenos os problemas ligados à seca. No nível de probabilidade de $75 \%$, o qual normalmente é referência para projetos de irrigação, verifica-se uma precipitação provável média anual de $72,2 \mathrm{~mm}$, no período chuvoso (novembro a março) este valor foi de $144 \mathrm{~mm}$ e no período seco (abril a outubro), $20,9 \mathrm{~mm}$. Com base neste tipo de análise, o projetista poderá dimensionar o sistema de irrigação ou o manejo desta, levando-se em conta esta informação, que é obtida assumindo-se um nível de probabilidade aceitável ou teoricamente viável. Ressalta-se que, no mês de julho, as estimativas não são confiáveis, haja vista a não adequacidade para esta série.

$\mathrm{Na}$ Figura 2 apresentam-se os valores de precipitação provável para períodos quinzenais. Utilizou-se a distribuição de probabilidade Gama por esta ter se apresentado de forma mais adequada para os referidos períodos. Observa-se tendência semelhante à apresentada para as precipitações mensais, tanto na primeira, quanto na segunda quinzena, verificando-se, no nível de probabilidade de $75 \%$, a precipitação quinzenal média anual é de $26 \mathrm{~mm}$, no período chuvoso, de $53,9 \mathrm{~mm}$ e no período seco, $6 \mathrm{~mm}$.

Na Figura 3, apresentam-se os valores de precipitação provável para períodos decendiais. Utilizou-se a distribuição de probabilidade Gama por esta ter se apresentado de forma mais adequada para os referidos períodos. Observa-se tendência semelhante à apresentada para as precipitações mensais e quinzenais, em que no nível de $75 \%$ de probabilidade, verifica-se uma precipitação média anual de $17,6 \mathrm{~mm}$, no período chuvoso, de $34,8 \mathrm{~mm}$ e no período seco, 5,4 $\mathrm{mm}$. Destaca-se que, do terceiro decêndio de abril até o segundo decêndio de setembro, as estimativas não são confiáveis, haja vista a não adequabilidade destas séries ao teste de KolmogorovSmirnov.

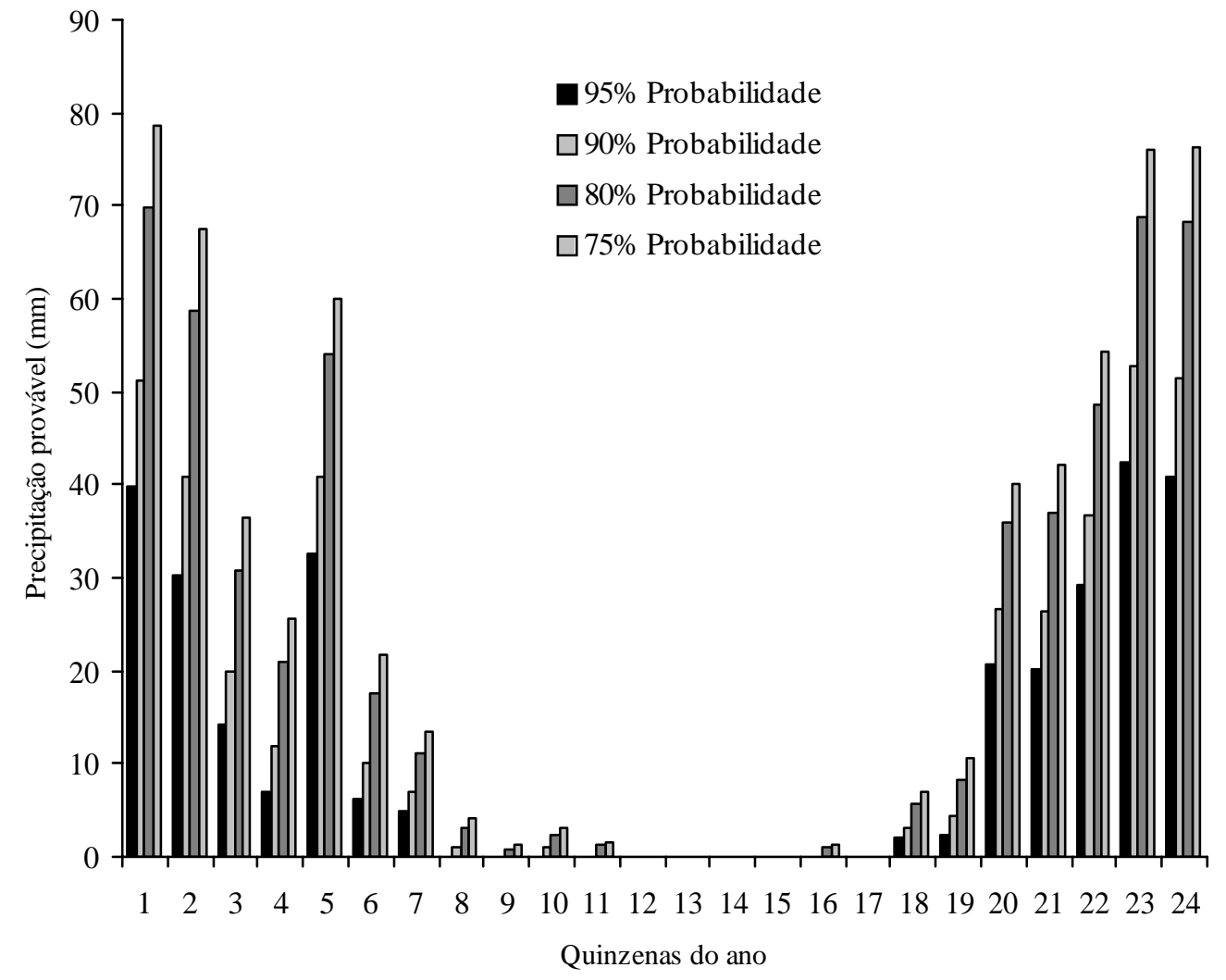

FIGURA 2 - Precipitação provável quinzenal segundo a distribuição de probabilidade Gama.

Ciênc. agrotec., Lavras, v. 31, n. 3, p. 842-850, maio/jun., 2007 


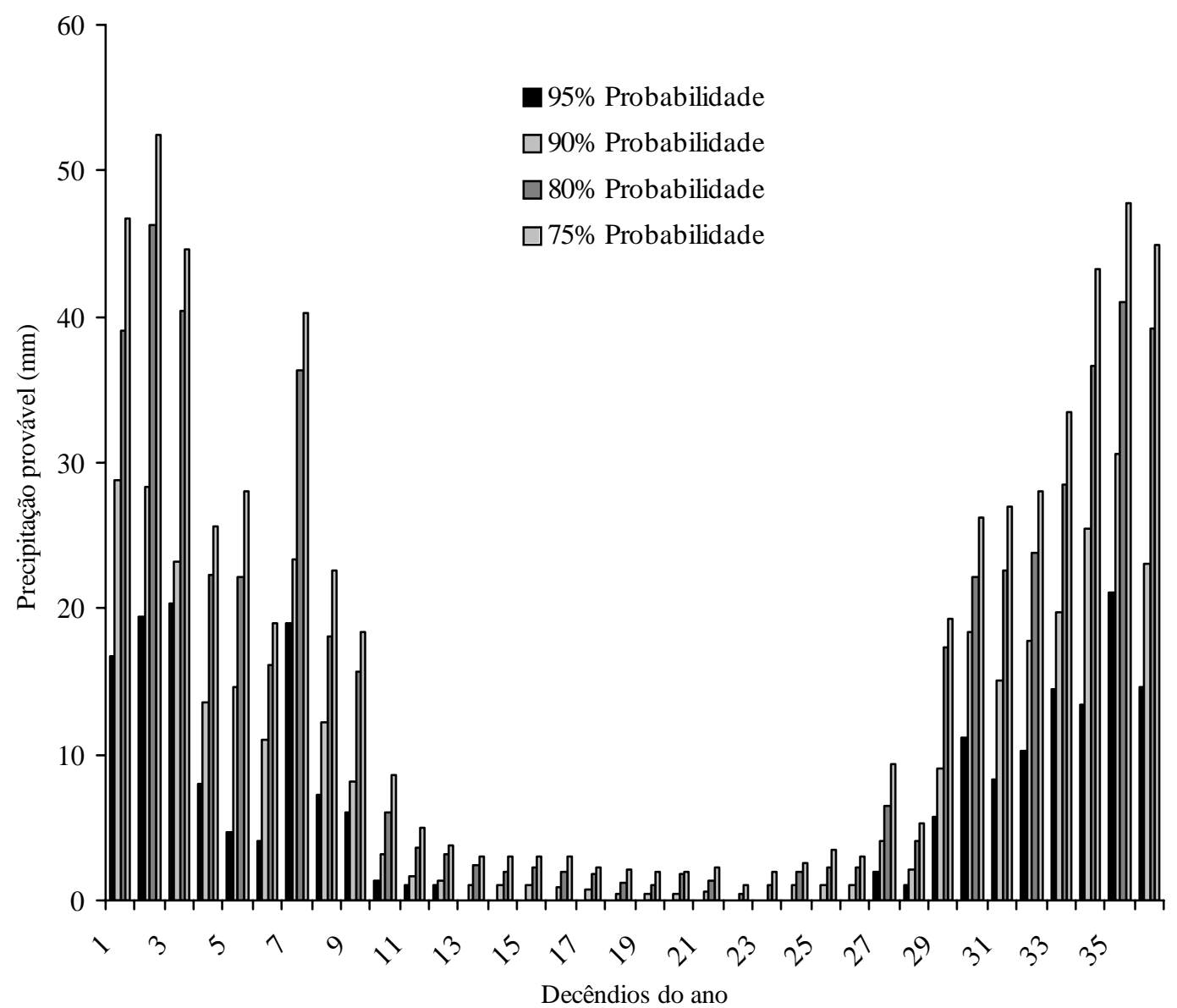

FIGURA 3 - Precipitação provável decendial segundo a distribuição de probabilidade Gama.

\section{CONCLUSÕES}

A distribuição de probabilidades Log-normal 3 parâmetros foi a que melhor se ajustou às séries históricas de precipitações mensais, enquanto para os períodos quinzenais e decendiais, destacou-se a distribuição Gama.

A região de estudo apresentou valores médios anuais de precipitação mensal, quinzenal e decendial consideráveis para o nível de probabilidade de $75 \%$, sendo respectivamente, de 72,2 mm, $26 \mathrm{~mm}$ e 17,6 mm, mostrando ser uma região onde provavelmente, os problemas ligados à seca são menores, e este aspecto pode ser levado em consideração para um melhor planejamento da agricultura irrigada.

\section{REFERÊNCIAS BIBLIOGRÁFICAS}

ANDRADE, C. L. T.; COELHO, E. F.; COUTO, L.; SILVA, E. L. Parâmetros de solo-água para engenharia de irrigação e ambiental. In: FARIA, M. A.; SILVA, E. L.; VILELA, L. A. A.; SILVA, A. M. Manejo de irrigação. Lavras: UFLA/ SBEA, 1998. p. 1-132.

ANTUNES, F. Z. Caracterização climática do Estado de Minas Gerais. Informe Agropecuário, Belo Horizonte, v. 12, n. 138 , p. $9-13,1986$.

BEIJO, L. A.; MUNIZ, J. A.; VOLPE, C. A.; PEREIRA, G. T. Estudo da precipitação máxima em Jaboticabal (SP) pela distribuição de Gumbel utilizando dois métodos de estimação dos parâmetros. Revista Brasileira de Agrometeorologia, Santa Maria, v. 11, n. 1, p. 141-147, 2003.

CASTRO NETO, P.; SILVEIRA, J. V. Precipitação provável para Lavras, região Sul de Minas Gerais, baseada na função de distribuição de probabilidade gama I: períodos mensais. Ciência e Prática, Lavras, v. 5, n. 2, p. 144-151, 1981a. 
CASTRO NETO, P.; SILVEIRA, J. V. Precipitação provável para Lavras, região Sul de Minas Gerais, baseada na função de distribuição de probabilidade gama II: períodos de quinze dias. Ciência e Prática, Lavras, v. 5, n. 2, p. $152-162,1981 b$

CASTRO NETO, P.; SILVEIRA, J. V. Precipitação provável para Lavras-MG, baseada na função de distribuição de probabilidade gama III: períodos de 10 dias. Ciência e Prática, Lavras, v. 7, n. 1, p. 58-65, 1983.

FRIZZONE, J. A. Análise de cinco modelos para cálculo da distribuição de precipitação na região de Viçosa - MG. 1979. 100 f. Dissertação (Mestrado) - Universidade Federal de Viçosa, Viçosa, 1979.

GOMIDE, R. L. Monitoramento para manejo da irrigação: instrumentação, automação e métodos. In: FARIA, M. A.; SILVA, E. L.; VILELA, L. A. A.; SILVA, A. M. Manejo de Irrigação. Lavras: UFLA/SBEA, 1998. p. 133-238.

HAAN, C. T. Statistical methods in hydrology. 2. ed. Ames: Iowa State University, 1979. 377 p.
LANNA, A. E. Elementos de estatística e probabilidades. In: TUCCI, C. E. M. (Org.). Hidrologia: ciência e aplicação. 2. ed. Porto Alegre: ABRH/UFRGS, 2001. p. 79-176.

RIGHETTO, A. M. Hidrologia e recursos hídricos. São Carlos: EESC/USP, 1998. 819 p.

SAMPAIO, S. C. et al. Precipitação provável para o município de Lavras-MG, região Sul de Minas Gerais, utilizando a distribuição Log-normal. Ciência e Agrotecnologia, Lavras, v. 23, n. 2, p. 382-389, abr./jun. 1999.

SILVA, A. M.; MELLO, C. R. de. Estudo hidrológico sobre o regime de escoamento de sub-bacias de drenagem para o reservatório de Camargos/CEMIG, Alto Rio Grande. Lavras: UFLA/FAEPE/CEMIG, 2005. 200 p.

WALPOLE, R. E.; MYERS, R. H. Probability and statistics for engineers and scientists. New York: Macmillan, 1978. $580 \mathrm{p}$. 